\title{
Hydrocephalus in children with ruptured cerebral arteriovenous malformation
}

\author{
*Sarah Stricker, MD, ${ }^{1}$ Grégoire Boulouis, MD, ${ }^{1,2,4}$ Sandro Benichi, MD, ${ }^{1}$ Florent Gariel, MD, ${ }^{1}$ \\ Lorenzo Garzelli, MD, ${ }^{1}$ Kevin Beccaria, MD, PhD, ${ }^{1,3}$ Anais Chivet, MD, ${ }^{1}$ \\ Timothee de Saint Denis, MD, ${ }^{1}$ Syril James, MD, ${ }^{1}$ Giovanna Paternoster, MD, ${ }^{1}$ \\ Michel Zerah, MD, PhD, ${ }^{1,2}$ Marie Bourgeois, MD, ${ }^{1}$ Nathalie Boddaert, MD, PhD, ${ }^{1,3}$ \\ Francis Brunelle, MD, PhD, ${ }^{1}$ Philippe Meyer, MD, ${ }^{1}$ Stephanie Puget, MD, PhD, ${ }^{1,3}$ \\ Olivier Naggara, MD, PhD, 1,2,4 and Thomas Blauwblomme, MD, PhD1,3
}

${ }^{1}$ APHP, Necker Hospital, Université de Paris; '2Department of Neuroradiology, INSERM U1266; 3Imagine Institute, INSERM U1163; and ${ }^{4}$ Interventional Neuroradiology Centre Hospitalier Sainte-Anne, Paris, France

OBJECTIVE Hydrocephalus is a strong determinant of poor neurological outcome after intracerebral hemorrhage (ICH). In children, ruptured brain arteriovenous malformations (bAVMs) are the dominant cause of ICH. In a large prospective cohort of pediatric patients with ruptured bAVMs, the authors analyzed the rates and predictive factors of hydrocephalus requiring acute external ventricular drainage (EVD) or ventriculoperitoneal shunt (VPS).

METHODS The authors performed a single-center retrospective analysis of the data from a prospectively maintained database of children admitted for a ruptured bAVM since 2002. Admission clinical and imaging predictors of EVD and VPS placement were analyzed using univariate and multivariate statistical models.

RESULTS Among 114 patients (mean age 9.8 years) with 125 distinct ICHs due to ruptured bAVM, EVD and VPS were placed for $55 / 125$ (44\%) hemorrhagic events and 5/114 patients (4.4\%), respectively. A multivariate nominal logistic regression model identified low initial Glasgow Coma Scale (iGCS) score, hydrocephalus on initial CT scan, the presence of intraventicular hemorrhage (IVH), and higher modified Graeb Scale (mGS) score as strongly associated with subsequent need for EVD (all $p<0.001$ ). All children who needed a VPS had initial hydrocephalus requiring EVD and tended to have higher mGS scores.

CONCLUSIONS In a large cohort of pediatric patients with ruptured bAVM, almost half of the patients required EVD and $4.4 \%$ required permanent VPS. Use of a low iGCS score and a semiquantitative mGS score as indicators of the IVH burden may be helpful for decision making in the emergency setting and thus improve treatment.

https://thejns.org/doi/abs/10.3171/2020.3.PEDS19680

KEYWORDS hydrocephalus; ventriculoperitoneal shunt; arteriovenous malformation; intracerebral hemorrhage; external ventricular drain; vascular disorders

$\mathrm{R}$ uptured brain arteriovenous malformations (bAVMs) are the main cause of pediatric intracerebral hemorrhage $(\mathrm{ICH}){ }^{1-3}$ Acute hydrocephalus secondary to intraventricular hemorrhage (IVH) or ventricle compression is a key therapeutic target at the acute phase to improve neurological outcome. ${ }^{4} \mathrm{CSF}$ diversion procedures, i.e., acute external ventricular drainage (EVD) and delayed ventriculoperitoneal shunt (VPS) placement, have been studied only in adult cohorts. ${ }^{5-11}$ These proce- dures are reported in $13 \%-53 \%$ and $1.9 \%-24.2 \%$ of cases, respectively, depending on the setting and underlying ICH etiology.

In pediatric ICH, data are scarce on the need for EVD and VPS, leading to uncertainty in acute decision making for CSF diversion and care stratification. We aimed to assess the rates and predictive factors of hydrocephalus requiring acute EVD and VPS in children with ICH after bAVM rupture.

ABBREVIATIONS AUC = area under the curve; bAVM = brain arteriovenous malformation; EVD = external ventricular drainage; ICH = intracerebral hemorrhage; iGCS = initial Glascow Coma Scale; IVH = intraventricular hemorrhage; KOSCHI = King's Outcome Scale for Childhood Head Injury; mGS = modified Graeb Scale; VPS = ventriculoperitoneal shunt.

SUBMITTED November 12, 2019. ACCEPTED March 10, 2020.

INCLUDE WHEN CITING Published online May 22, 2020; DOI: 10.3171/2020.3.PEDS19680.

* S.S. and G.B. contributed equally to this work. 


\section{Methods}

STROBE (Strengthening the Reporting of Observational Studies in Epidemiology) guidelines were followed..$^{12}$ Because the investigation was a noninterventional retrospective study of routinely acquired data, the requirement for written informed consent from all participating patients was waived, and patients and their guardians were informed that they could oppose the use of their health-related data for research purposes. The institutional review board (CPP Ile de France III; 18062012) approved the collection of data into the dedicated database.

\section{Study Design and Data Extraction}

Based on the previously described registry of children presenting with ICH at the pediatric hospital of our institution (Necker Enfants Malades, Paris, France), ${ }^{2}$ patients $<18$ years old who were treated for ruptured bAVM at our institution since 2002 were identified. Clinical data included age at event, sex, and initial Glasgow Coma Scale (iGCS) score and were retrospectively completed with the help of a data warehouse. ${ }^{13}$ Clinical outcome, assessed 18 months after ICH, was defined as favorable for King's Outcome Scale for Childhood Head Injury (KOSCHI) scores of $\geq 5$ and severely disabled for KOSCHI scores of $<4 .{ }^{14}$ Six patients with follow-up periods of $<18$ months were excluded from outcome analysis.

Imaging characteristics included bAVM angioarchitecture, i.e., Spetzler-Martin grade, associated aneurysms, deep venous drainage, deep AVM localization, and ICH volume (ml). IVH extent was assessed using the modified Graeb Scale (mGS) score, which ranges from 0 to 32 and is based on the distribution and amount of IVH and associated ventricular dilatation. ${ }^{15}$ Temporal horn dilatation was defined as a diameter larger than $5 \mathrm{~mm}$. According to the institution's practice, which remained unchanged during the study period, the placement of an EVD was indicated to control intracranial pressure in case of either IVH or signs of elevated intracranial pressure on initial imaging, such as shift of midline structures, herniation, or cerebral edema. The EVD was leveled at 15 or $20 \mathrm{~mm} \mathrm{H}_{2} \mathrm{O}$ and was removed when the drained CSF was free of blood and IVH was cleared on follow-up CT.

\section{Statistical Analysis}

Statistical analyses were performed using JMP Pro version 14.0 (SAS Institute Inc., 2019). Differences in bleeding events with and without the need for EVD placement were studied in univariate analyses performed using appropriate tests for each variable category. Those variables associated with EVD placement $(\mathrm{p}<0.1)$ were entered in a multivariate nominal logistic regression model, while nonsignificant variables were backward eliminated. Before performance of the univariable tests, it was prespecified that in the multivariable model age and mGS score would be adjusted independently of their association with EVD placement found in the univariable tests. Two-sided $\mathrm{p}$ values $<0.05$ were considered statistically significant.

\section{Results}

\section{Patient Characteristics}

Clinical and radiological characteristics of 114 included patients with $125 \mathrm{ICHs}$ are summarized in Table 1. The distribution of Spetzler-Martin grades was as follows: $21 \%$ grade I, $42 \%$ grade II, $23 \%$ grade III, $10 \%$ grade IV, and $4 \%$ grade V. At the acute phase, a surgical evacuation of

TABLE 1. Clinical and radiological characteristics of 114 patients with ruptured bAVM

\begin{tabular}{|c|c|c|c|c|}
\hline \multirow[b]{2}{*}{ Variable } & \multirow[b]{2}{*}{ Patients $(n=114)^{*}$} & \multicolumn{2}{|c|}{ Hemorrhagic Events $(n=125)$} & \multirow[b]{2}{*}{$p$ Value } \\
\hline & & $\operatorname{EVD}(n=55)$ & No EVD $(n=70)$ & \\
\hline Male sex & $64(56 \%)$ & $32(58.2 \%)$ & $36(51.4 \%)$ & 0.47 \\
\hline Age in yrs & $9.6(3.7)$ & $10.7(3.6)$ & $9.1(3.9)$ & 0.009 \\
\hline iGCS score & $11.4(4.0)$ & $9.7(4.0)$ & $13.2(3.0)$ & $<0.0001$ \\
\hline iGCS score <9 & $34(30 \%)$ & $26(47.3 \%)$ & $9(12.9 \%)$ & $<0.0001$ \\
\hline IVH & $68(60 \%)$ & $52(95 \%)$ & $22(32 \%)$ & $<0.0001$ \\
\hline IVH only & $10(9 \%)$ & $4(7.3 \%)$ & $9(12.9 \%)$ & 0.38 \\
\hline mGS score & $10.7(11.3)$ & $18.5(10.2)$ & $4.4(7.6)$ & $<0.0001$ \\
\hline bAVM aneurysm & $37(32 \%)$ & $25(45.5 \%)$ & $15(21.7 \%)$ & 0.007 \\
\hline Superficial bAVM & $47(41 \%)$ & $16(29.1 \%)$ & $37(52.9 \%)$ & 0.008 \\
\hline Any deep drainage & $57(50.4 \%)$ & $34(61.8 \%)$ & $30(43.5 \%)$ & 0.032 \\
\hline $\mathrm{ICH}$ volume, $\mathrm{ml}$ & $20.0(16.4)$ & $20.2(17.4)$ & $19(15.9)$ & 0.68 \\
\hline Temporal horn dilatation & $44(38.6 \%)$ & $41(74.5 \%)$ & $8(11 \%)$ & $<0.0001$ \\
\hline \multicolumn{5}{|l|}{ Outcome† } \\
\hline Favorable outcome & $65 / 108(60 \%)$ & $26 / 50(52 \%)$ & $39 / 58(67 \%)$ & 0.12 \\
\hline Severe disability or worse & $15 / 108(14 \%)$ & $10 / 50(20 \%)$ & $5 / 58(5 \%)$ & 0.03 \\
\hline
\end{tabular}


TABLE 2. Multivariable model analysis of predictive factors for EVD placement after ruptured bAVM

\begin{tabular}{lccc}
\hline \multicolumn{1}{c}{ Variable } & Adjusted & & \\
& OR & $95 \% \mathrm{Cl}$ & p Value \\
\hline Age at event (per yr) & 1.16 & $0.98-1.37$ & 0.07 \\
\hline iGCS score (per point) & 0.74 & $0.63-0.88$ & $\mathbf{0 . 0 0 1 8}$ \\
\hline Dilated temporal horn(s) & 7.45 & $2.03-27.38$ & $\mathbf{0 . 0 0 2}$ \\
\hline mGS score (per point) & 1.16 & $1.08-1.25$ & $<\mathbf{0 . 0 0 0 1}$ \\
\hline Aneurysm (yes) & 3.42 & $0.85-13.85$ & 0.07 \\
\hline bAVM location (superficial vs deep) & 0.75 & $0.19-2.89$ & 0.67 \\
\hline Any deep drainage (yes) & 0.63 & $0.16-2.38$ & 0.49 \\
\hline
\end{tabular}

Boldface type indicates statistical significance.

the parenchymal hematoma and a targeted endovascular embolization was performed in, respectively, 53 and 42 patients, and 4 patients died during initial hospitalization.

\section{Acute Hydrocephalus and Predictive Factors for EVD Placement}

EVD was required in 55/125 (44\%) of the hemorrhagic events and placed after a median of 0 days (IQR $0-1$ ) following initial imaging, for an average duration of $5.5 \pm 7.6$ days. Five of the 114 patients (4.4\%) required VPS, all of whom had an acute EVD placement.

In univariate analysis, older age, lower iGCS score, IVH, higher mGS score, temporal horn dilatation, bAVMrelated aneurysms, and deeply located bAVMs were associated with EVD (Table 1). After adjustment in the multivariable analysis (Table 2), mGS and iGCS were the most powerful predictors for EVD placement (Fig. 1), and bAVM-related aneurysms tended toward significance. The AUC for EVD placement in the fully adjusted multivariate model was 0.95 (95\% CI [1000 bootstraps] 0.91-0.98), indicating excellent predictive ability (Fig. 2). The results were similar after further adjustment for bleed recurrence. No relationship between the Spetzler-Martin grading and the need for EVD was found (chi square 4.497, $\mathrm{p}=0.34)$. All children requiring VPS required an EVD and had higher mGS scores (mGS scores with vs those without VPS 19.6 vs 10.2, p = 0.06). On follow-up, there was no detectable difference in favorable outcome among children with and those without EVD treatment (52\% vs $67 \%$ with $\mathrm{KOSCHI} \geq 5, \mathrm{p}=0.25$ ), but those with EVD were more frequently severely disabled or worse (KOSCHI score $<4$ in $19 \%$ with EVD vs $8 \%$ without, $\mathrm{p}=0.04)$.

\section{Discussion}

Our study provides a data-driven framework aimed at guiding acute decision making with regard to the need for EVD placement and long-term shunt dependence in the setting of ruptured bAVM in pediatric patients.

In the present study of children with bAVM-related ICH, EVD placement was required in $44 \%$ and VPS placement in $4.4 \%$ of patients, rates comparable to those found in studies of adult patients. ${ }^{5,8,9,11}$ Significant risk factors for EVD placement were iGCS score and the presence of IVH and hydrocephalus on initial brain imaging. In addition, we found that the main determinant for EVD placement was the IVH burden assessed by mGS. This was true independently of baseline hydrocephalus. However, because this was a retrospective study without predetermined criteria for EVD placement, the independence of the predictive factors can only be assumed. Also, the establishment of a predictive score was impossible due to the limited statistical power of the study owing to the rarity of pediatric bAVM. As a representative example, in a 10-year-old child with an iGCS score of 13, the probabilities for EVD placement with mGS scores of $0,10,20$, and 30 would be $6.5 \%, 23 \%, 57 \%$, and $86 \%$, respectively. As the median delay between initial CT and EVD placement was $<24$ hours, our results suggest the importance of prompt transfer to a neurosurgical ICU for these patients. In the management of children without symptomatic hydrocephalus, no beneficial effect of EVD was found; nevertheless, we advocate close observation of these patients, especially in cases with very extensive IVH and uncertainty regarding low-threshold EVD placement. The study results highlight the important findings that EVD placement in the emergency setting does not increase the risk associated with

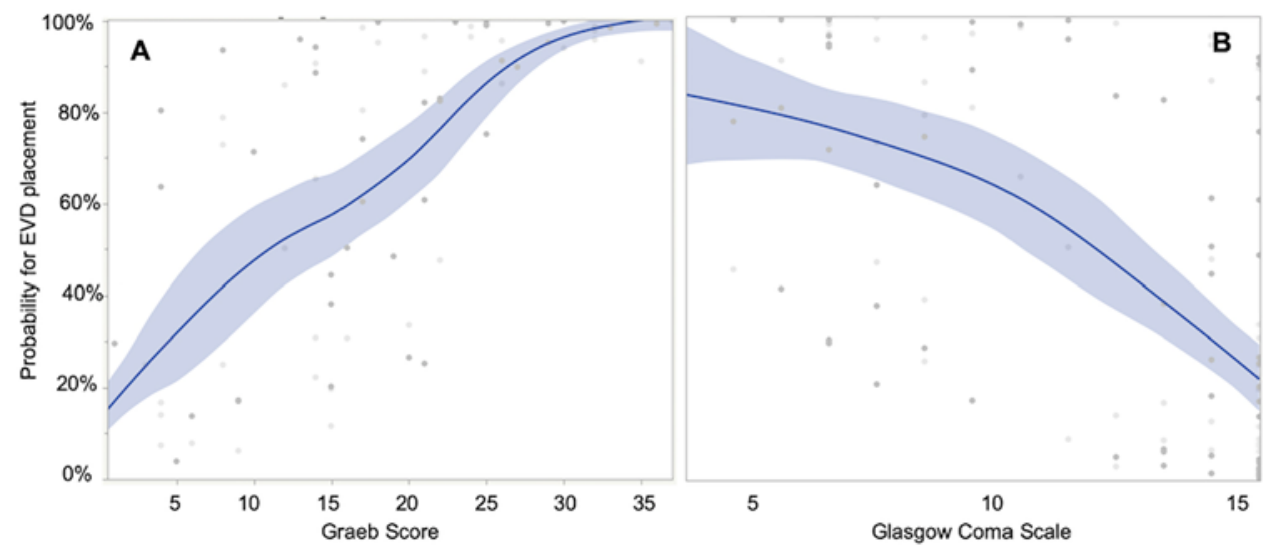

FIG. 1. Probability of EVD, in the fully adjusted model, plotted against the mGS score (GS; $A$ ) and the iGCS score (GCS; B). Figure is available in color online only. 


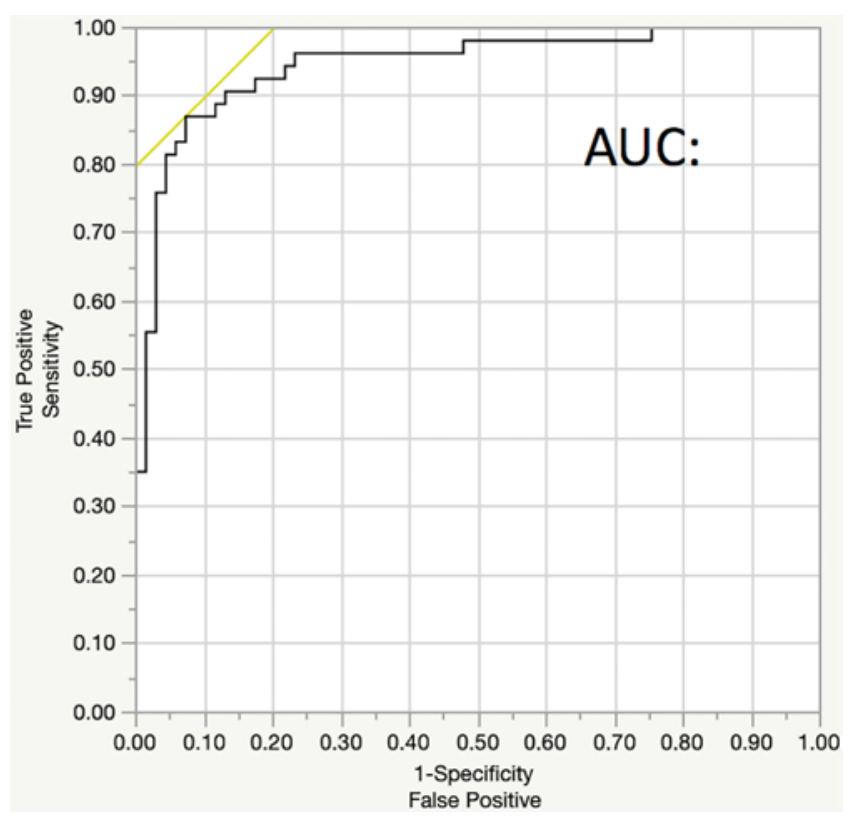

FIG. 2. AUC for EVD placement in the model adjusted for male sex, age at event, iGCS score, dilated temporal horn(s), mGS score, AVM-related aneurysm, AVM location, and any deep drainage. Figure is available in color online only.

VPS insertion or the risk of infection. Thus indications for EVD placement after rupture of pediatric bAVM can be kept wide, thereby helping to optimize the management of intracranial pressure.

Baseline ICH volume, although not predictive of acute EVD placement, was among the angioarchitectural characteristics of the bAVM-associated aneurysms that tended to increase the probability of EVD placement. This finding has already been demonstrated in an adult population. ${ }^{5}$ Aneurysms may have been responsible for subsequent worsening of ICH volume and IVH load, likely explaining their higher prevalence in children requiring EVD. Hence, identification of an associated aneurysm responsible for the ICH may have therapeutic implications because these aneurysms may be associated with higher morbidity through a higher incidence of hydrocephalus requiring EVD placement.

Our data also reinforced the finding that children who needed EVD placement were more frequently severely disabled, which has significant clinical implications for hydrocephalus and EVD placement after pediatric bAVM rupture. Hydrocephalus and the resulting elevated intracranial pressure in the setting of $\mathrm{ICH}$ are considerable sources of neurological morbidity. ${ }^{16}$ Further research is needed on the impact of hydrocephalus, including the influence of methods used for treatment on long-term cognitive development in pediatric patients with ruptured bAVMs.

\section{Conclusions}

After bAVM rupture in children, nearly half will require an acute EVD placement. Significant risk factors for EVD placement were high mGS score and low iGCS score. As EVD placement does not increase the risk for VPS, the indications for EVD placement after rupture of bAVM can be kept wide, which helps to optimize the available options for intracranial pressure management.

\section{Acknowledgments}

We thank the Imagine Institute, and notably Dr. N. Garcelon, for help with the Dr Warehouse software.

\section{References}

1. Ding D, Starke RM, Kano H, et al. International multicenter cohort study of pediatric brain arteriovenous malformations. Part 1: Predictors of hemorrhagic presentation. J Neurosurg Pediatr. 2017;19(2):127-135.

2. Guédon A, Blauwblomme T, Boulouis G, et al. Predictors of outcome in patients with pediatric intracerebral hemorrhage: development and validation of a modified score. Radiology. 2018;286(2):651-658.

3. Nair AP, Kumar R, Mehrotra A, et al. Clinical, radiological profile and outcome in pediatric Spetzler-Martin grades I-III arteriovenous malformations. Childs Nerv Syst. 2012;28(4):593-598.

4. Diringer MN, Edwards DF, Zazulia AR. Hydrocephalus: a previously unrecognized predictor of poor outcome from supratentorial intracerebral hemorrhage. Stroke. 1998;29(7):1352-1357.

5. Gross BA, Lai PMR, Du R. Hydrocephalus after arteriovenous malformation rupture. Neurosurg Focus. 2013;34(5):E11.

6. Hughes JD, Puffer R, Rabinstein AA. Risk factors for hydrocephalus requiring external ventricular drainage in patients with intraventricular hemorrhage. J Neurosurg. 2015;123(6):1439-1446.

7. Hwang BY, Bruce SS, Appelboom G, et al. Evaluation of intraventricular hemorrhage assessment methods for predicting outcome following intracerebral hemorrhage. J Neurosurg. 2012;116(1):185-192.

8. Murthy SB, Merkler AE, Omran SS, et al. Outcomes after intracerebral hemorrhage from arteriovenous malformations. Neurology. 2017;88(20):1882-1888.

9. Torné R, Rodríguez-Hernández A, Arikan F, et al. Posterior fossa arteriovenous malformations: significance of higher incidence of bleeding and hydrocephalus. Clin Neurol Neurosurg. 2015;134:37-43.

10. Weinstein R, Ess K, Sirdar B, et al. Primary intraventricular hemorrhage: clinical characteristics and outcomes. J Stroke Cerebrovasc Dis. 2017;26(5):995-999.

11. Ye Z, Ai X, Hu X, et al. Clinical features and prognostic factors in patients with intraventricular hemorrhage caused by ruptured arteriovenous malformations. Medicine (Baltimore). 2017;96(45):e8544.

12. von Elm E, Altman DG, Egger M, et al. The Strengthening the Reporting of Observational Studies in Epidemiology (STROBE) statement: guidelines for reporting observational studies. Lancet. 2007;370(9596):1453-1457.

13. Garcelon N, Neuraz A, Salomon R, et al. A clinician friendly data warehouse oriented toward narrative reports: Dr. Warehouse. J Biomed Inform. 2018;80:52-63.

14. Calvert S, Miller HE, Curran A, et al. The King's Outcome Scale for Childhood Head Injury and injury severity and outcome measures in children with traumatic brain injury. Dev Med Child Neurol. 2008;50(6):426-431.

15. Morgan TC, Dawson J, Spengler D, et al. The Modified Graeb Score: an enhanced tool for intraventricular hemorrhage measurement and prediction of functional outcome. Stroke. 2013;44(3):635-641. 
16. Blauwblomme T, Bourgeois M, Meyer P, et al. Long-term outcome of 106 consecutive pediatric ruptured brain arteriovenous malformations after combined treatment. Stroke. 2014;45(6):1664-1671.

\section{Disclosures}

The authors report no conflict of interest concerning the materials or methods used in this study or the findings specified in this paper.

\section{Author Contributions}

Conception and design: Blauwblomme, Stricker, Boulouis, Benichi, Naggara. Acquisition of data: Blauwblomme, Stricker, Boulouis, Gariel, Garzelli, Beccaria, Chivet, de Saint Denis, James, Paternoster, Zerah, Bourgeois, Boddaert, Brunelle,
Meyer, Puget, Naggara. Analysis and interpretation of data: Blauwblomme, Stricker, Boulouis, Benichi, Zerah, Boddaert, Brunelle, Meyer, Puget, Naggara. Drafting the article:

Blauwblomme, Stricker, Boulouis, Naggara. Critically revising the article: Blauwblomme, Stricker, Boulouis, Benichi, Naggara. Reviewed submitted version of manuscript: Blauwblomme, Stricker, Boulouis, Benichi, Naggara. Approved the final version of the manuscript on behalf of all authors: Blauwblomme. Statistical analysis: Stricker, Boulouis. Administrative/techni$\mathrm{cal} /$ material support: Stricker, Boulouis. Study supervision: Blauwblomme, Benichi, Naggara.

\section{Correspondence}

Thomas Blauwblomme: Necker Hospital, Université de Paris, France. thomas.blauwblomme@aphp.fr. 